\title{
Differential equations characterizing space curves of constant breadth and their solutions
}

\author{
Tuba Agirman Aydin ${ }^{1}$ and Mehmet Sezer ${ }^{2}$ \\ ${ }^{1}$ Faculty of education, Bayburt University, Bayburt, Turkey \\ ${ }^{2}$ Department of Mathematics, Celal Bayar University, Manisa, Turkey
}

Received: 30 June 2017, Accepted: 23 October 2017

Published online: 13 March 2018.

\begin{abstract}
In this study, we first show that the system of Frenet-like differential equation [14] characterizing space curves of constant breadth $[10,11,13]$ is equivalent to a third order, linear, differential equation with variable coefficients. Then, by using Taylor matrix method based on collocations points [7,8], we obtain the set of solution of the mentioned differential equation under the initial conditions in terms of Taylor polynomials. Furthermore, we discuss that the obtained results are useable to determine curves of constant breadth.
\end{abstract}

Keywords: Space curves of constant breadth, Taylor matrix method, linear differential equations.

\section{Introduction}

Curves of constant breadth firstly were introduced by L. Euler [3], in 1778. F. Reuleaux [12] gave the obtaining method some curves of constant breadth in 1963 and led to be used in kinematics of machinery. So far, in mathematics, many geometers have obtained only geometric properties of the plane curves of constant breadth, but have had a few study on space curves of constant breadth [2, 6, 9]. M. Fujivara had obtained a problem to determine whether there exist "space curve of constant breadth" or not and he defined "breadth" for space curves and obtained these curves on a surface of constant breadth [4]. Having been used the basic concepts [5, 11] concerned with the space curves of constant breadth, a integral characterization of these curves [14] has been obtained and a criterion for these curves has been determined [13]. Also the curves of constant breadth were extented to the $\mathrm{E}^{4}$ - space and some characterizations were obtained [11]. In addition, Akdoğan and Mağden [1] were extended this kind of curves to $\mathrm{E}^{n}$ space and some characterizations were obtained. Studies on these curves are going on nowadays, currently. These curves are used in the kinematics of machinary, engineering and com design.

In this study, our first aim, by means of (2), to establish differential equations with unknowns $\lambda, \mu$ and $\delta$ discribing a curve of constant breadth. The second is to find the approximate solutions of these differential equations under the initial conditions in terms of Taylor polynomials.

\section{The space curves of constant breadth}

The base of our study is based on the following consepts for space curves of constant breadth, which are presented by Ö. Köse $[9,10]$ and M. Sezer [13, 14]. 
Let $(\mathrm{C})$ be a space curve in the class $\Gamma$ having parallel tangents $t$ and $t^{*}$ in opposite directions at the opposite points $\alpha$ and $\alpha^{*}$ of the curve [10]. If the chord joining the opposite points of $(\mathrm{C})$ is a double-normal, then $(\mathrm{C})$ has constant breadth, and conversely, if (C) is a curve of constant breadth, then every normal of (C) is a double-normal. A simple closed curve (C) of constant breadth having parallel tangents in opposite directions at opposite points may be represented by the equation

$$
\alpha^{*}(s)=\alpha(s)+\lambda(s) \mathbf{t}+\mu(s) \mathbf{n}+\delta(s) \mathbf{b}
$$

where $\alpha$ and $\alpha^{*}$ are opposite points, and $\mathbf{t}, \mathbf{n}, \mathbf{b}$ denote the unite tangent, principal normal, binormal at a generic point $\alpha$, respectively. Here sdenotes the arc length of (C) and the curvature of the curve is

$$
\lim _{\Delta s \rightarrow 0} \frac{\Delta \phi}{\Delta s}=\frac{d \phi}{d s}=\varkappa(s)
$$

where $\Delta \phi$ is the angle of contengency. Here $\phi$ denotes the angle between tangent of the curve (C) at the point $\alpha(s)$ and a given fixed direction. Also it is clear that

$$
\phi(s)=\int_{0}^{s} \varkappa(s) d s
$$

In this case a pair of opposite points of the curve is $\left(\alpha^{*}(s), \alpha(s)\right)$ for s. On the other hand, the coefficients $\lambda, \mu$ and $\delta$ may be obtained by the

$$
\begin{aligned}
& \lambda^{\prime}=\mu-g(\phi) \\
& \mu^{\prime}=-\lambda+\rho \tau \delta \\
& \delta^{\prime}=-\rho \tau \mu
\end{aligned}
$$

which is the system describing the curve (1). $g(\phi)=\rho+\rho^{*}$ and,

$$
\rho=\frac{1}{\varkappa} \text { and } \rho^{*}=\frac{1}{\varkappa^{*}}
$$

denote the radii of curvatures $\alpha(s)$ and $\alpha^{*}(s)$, respectively. Here (') denotes the differentiation with respect to $\phi$. Furthermore the distance $\mathrm{d}$ between the opposite points $\alpha$ and $\alpha^{*}$ is the breadth of the curve and is constant, that is,

$$
R^{2}=\|d\|^{2}=\lambda^{2}+\mu^{2}+\delta^{2}=\text { constant }
$$

Also, the vector $\mathbf{d}=\lambda(\phi) \mathbf{t}+\mu(\phi) \mathbf{n}+\delta(\phi) \mathbf{b}$ is the double normal of the curve (C) of constant breadth.

\section{Differential equations for the space curves of constant breadth}

In this section, we establish differential equations with unknowns $\lambda, \mu$ and $\delta$ characterizing the curves of constant breadth. First, it is clear that

$$
\mu=\lambda^{\prime}+g
$$

On the other hand, by using the second equation of the system (2) we obtain the following differential equation:

$$
\delta=\frac{1}{\rho \tau} \mu^{\prime}+\frac{1}{\rho \tau} \lambda .
$$


By using the derivative of the equation (3), we obtain the following differential equation:

$$
\delta=\frac{1}{\rho \tau} \lambda^{\prime \prime}+\frac{1}{\rho \tau} \lambda+\frac{1}{\rho \tau} \mathrm{g}^{\prime} .
$$

Also, it is clear that in the third equation of the system (2)

$$
\mu=-\frac{1}{\rho \tau} \delta^{\prime}
$$

Here, by using the equality of the equations (3) and (6) following equation is obtained

$$
\lambda^{\prime}+\frac{1}{\rho \tau} \delta^{\prime}+\mathrm{g}=0
$$

Finally, by using derivative of the equation (5), while $\mathrm{F}$ as follows

$$
F=(\rho \tau) g^{\prime \prime}-(\rho \tau)^{\prime} g^{\prime}+(\rho \tau)^{3} g
$$

we obtain the third order, linear, differential equation with variable coefficients as follows

$$
(\rho \tau) \lambda^{\prime \prime \prime}-(\rho \tau)^{\prime} \lambda^{\prime \prime}+(\rho \tau)\left(1+(\rho \tau)^{2}\right) \lambda^{\prime}-(\rho \tau)^{\prime} \lambda=F .
$$

As a result, it is clearly seen that the system (2) characterizing the space curves of constant breadth can be reduced to the linear differential equation (8). Furthermore, we can write this equation in the general form

$$
\sum_{\mathrm{k}=0}^{\mathrm{m}} \mathrm{Q}_{\mathrm{k}}(\phi) \lambda^{(\mathrm{k})}(\phi)=\mathrm{F}(\phi), \mathrm{m}=2,3, \ldots
$$

where $Q_{k}(\phi)$ are continuous functions of the expression $(\rho \tau)$.

\section{Taylor matrix method}

In this section, to obtain the Taylor polynomial solution of the differential equation defined by

$$
\sum_{\mathrm{k}=0}^{3} \mathrm{Q}_{\mathrm{k}}(\mathrm{s}) \lambda^{(\mathrm{k})}(\mathrm{s})=\mathrm{F}(\mathrm{s}), 0 \leq \mathrm{s} \leq \mathrm{b}
$$

Near the point $s=0$, under the initial conditions

$$
\begin{aligned}
\lambda(0) & =\lambda_{0} \\
\lambda^{\prime}(0) & =\lambda_{1} \\
\lambda^{\prime \prime}(0) & =\lambda_{2}
\end{aligned}
$$

we develop the Taylor matrix method based on collocation points, which is given by Sezer et al. [7, 8, 13, 14 ] Where $\lambda_{0}, \lambda_{1}, \lambda_{3}$ and $b$ appropriate constants.

Firstly, let us assume that the desired solution $\lambda(\mathrm{s})$ can be expanded to Taylor series about $\mathrm{s}=0$ in the form, for $\mathrm{N} \geq 3$ 


$$
\lambda(\mathrm{s})=\lambda_{\mathrm{N}}(\mathrm{s})=\sum_{\mathrm{n}=0}^{\mathrm{N}} \mathrm{a}_{\mathrm{n}} \mathrm{s}^{\mathrm{n}}, 0 \leq \mathrm{s} \leq \mathrm{b} .
$$

Where $a_{n}(n=0,1, \ldots, N)$ are the coefficients to be determined.

Now, we can convert the truncated Taylor series solution $\lambda(s)$ defined by (11) and its derivatives $\lambda^{(k)}(s), k=0,1,2,3$ to matrix forms, for $n=0,1, \ldots, N$,

$$
\lambda(s)=S(s) A
$$

and

$$
\lambda^{(k)}(s)=S^{(k)}(s) A, k=0,1,2,3, \ldots
$$

where

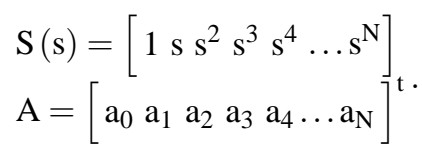

Also, it is clear that the relation between the matrix $S(s)$ and its derivative $S^{\prime}(s)$ is

$$
\mathrm{S}^{\prime}(\mathrm{s})=\mathrm{S}(\mathrm{s}) \mathrm{B}
$$

Where

$$
B=\left[\begin{array}{ccccccccc}
0 & 1 & 0 & 0 & 0 & \ldots & 0 & 0 & 0 \\
0 & 0 & 2 & 0 & 0 & \ldots & 0 & 0 & 0 \\
0 & 0 & 0 & 3 & 0 & \ldots & 0 & 0 & 0 \\
0 & 0 & 0 & 0 & 4 & \ldots & 0 & 0 & 0 \\
\vdots & \vdots & \vdots & \vdots & \vdots & \vdots & \vdots & \vdots & \vdots \\
0 & 0 & 0 & 0 & 0 & \ldots & n-2 & 0 & 0 \\
0 & 0 & 0 & 0 & 0 & \ldots & 0 & n-1 & 0 \\
0 & 0 & 0 & 0 & 0 & \ldots & 0 & 0 & n \\
0 & 0 & 0 & 0 & 0 & \ldots & 0 & 0 & 0
\end{array}\right]
$$

By repeating this process, we get the matrix relation as follows:

$$
\begin{aligned}
S^{\prime}(s) & =S(s) B \\
S^{\prime \prime}(s) & =S^{\prime}(s) B=S(s) B^{2} \\
S^{\prime \prime \prime}(s) & =S^{\prime \prime}(s) B=S(s) B^{3} \\
\vdots & \\
S^{(k)}(s) & =S(s) B^{k}, \quad k=0,1,2,3, \ldots
\end{aligned}
$$

From the matrix relations (11) and (12), it follows that

$$
\lambda^{(k)}(s) \cong S^{(k)}(s) A=S(s) B^{k} A, k=0,1,2,3 .
$$


We now ready to construct the fundamental corresponding to equation (9). For this purpose, by substituting the matrix relation (13) into equation (9) and by using the collocation points defined by

$$
s_{i}=\frac{b}{N} i, \quad i=0,1, \ldots, N
$$

we get the system of the matrix equations

$$
\left\{\sum_{\mathrm{k}=0}^{3} \mathrm{P}_{\mathrm{k}}\left(\mathrm{s}_{i}\right) \mathrm{S}\left(\mathrm{s}_{i}\right) \mathrm{B}^{\mathrm{k}}\right\} \mathrm{A}=\mathrm{f}\left(\mathrm{s}_{i}\right)
$$

or briefly the fundamental matrix equation

$$
\left\{\sum_{\mathrm{k}=0}^{3} \mathrm{P}_{\mathrm{k}} \mathrm{SB}^{\mathrm{k}}\right\} \mathrm{A}=\mathrm{F}
$$

where

$$
\begin{gathered}
P_{k}=\left[\begin{array}{cccccc}
P_{k}\left(s_{0}\right) & 0 & 0 & \ldots & 0 & 0 \\
0 & P\left(s_{1}\right) & 0 & \ldots & 0 & 0 \\
0 & 0 & P_{k}\left(s_{2}\right) & \ldots & 0 & 0 \\
\vdots & \vdots & \vdots & \vdots & \vdots & \vdots \\
0 & 0 & 0 & \ldots & P_{k}\left(s_{n-1}\right) & 0 \\
0 & 0 & 0 & \ldots & 0 & P_{k}\left(s_{N}\right)
\end{array}\right] \\
\mathrm{S}=\left[\begin{array}{c}
\mathrm{S}\left(\mathrm{s}_{0}\right) \\
\mathrm{S}\left(\mathrm{s}_{1}\right) \\
\vdots \\
\mathrm{S}\left(\mathrm{s}_{\mathrm{N}}\right)
\end{array}\right]=\left[\begin{array}{ccccc}
1 & \mathrm{~s}_{0} & \mathrm{~s}_{0}{ }^{2} & \ldots & \mathrm{s}_{0}{ }^{\mathrm{N}} \\
1 & \mathrm{~s}_{1} & \mathrm{~s}_{1}{ }^{2} & \ldots & \mathrm{s}_{1}{ }^{\mathrm{N}} \\
\vdots & \vdots & \vdots & \ddots & \vdots \\
1 & \mathrm{~s}_{\mathrm{N}} & \mathrm{s}_{\mathrm{N}}{ }^{2} & \ldots & \mathrm{s}_{\mathrm{N}}{ }^{\mathrm{N}}
\end{array}\right] \\
\mathrm{F}=\left[\begin{array}{c}
\mathrm{f}\left(\mathrm{s}_{0}\right) \\
\mathrm{f}\left(\mathrm{s}_{1}\right) \\
\vdots \\
\mathrm{f}\left(\mathrm{s}_{\mathrm{N}}\right)
\end{array}\right], \quad \mathrm{A}=\left[\begin{array}{c}
\mathrm{a}_{0} \\
\mathrm{a}_{1} \\
\vdots \\
\mathrm{a}_{\mathrm{N}}
\end{array}\right] .
\end{gathered}
$$

Hence equation (14) can be written in the form

$$
W A=F \text { or }[W ; F], W=\left[w_{p q}\right], p, q=0,1, \ldots, N
$$

where

$$
\begin{gathered}
\mathrm{W}=\left[\mathrm{w}_{\mathrm{pq}}\right]=\sum_{\mathrm{k}=0}^{3} \mathrm{P}_{\mathrm{k}} \mathrm{SB}^{\mathrm{k}}, \\
\mathrm{B}^{0}=\left[\begin{array}{cccc}
1 & 0 & \cdots & 0 \\
0 & 1 & \cdots & 0 \\
\vdots & \vdots & \ddots & \vdots \\
0 & 0 & \cdots & 1
\end{array}\right] .
\end{gathered}
$$


On the other hand, we can obtain the following matrix forms for the initial conditions (10), by means of the relation (13);

$$
\begin{gathered}
\lambda(0)=\lambda_{0} \Rightarrow S(0) B^{0} A=\lambda_{0} \\
\lambda^{\prime}(0)=\lambda_{1} \Rightarrow S(0) B A=\lambda_{1} \\
\lambda^{\prime \prime}(0)=\lambda_{2} \Rightarrow S(0) B^{2} A=\lambda_{2}
\end{gathered}
$$

or briefly

$$
U_{i} A=\left[\lambda_{i}\right] \Rightarrow\left[U_{i} ; \lambda_{i}\right], \quad i=0,1,2
$$

where

$$
\mathrm{U}_{\mathrm{i}}=\left[\begin{array}{llll}
\mathrm{u}_{\mathrm{i} 0} & \mathrm{u}_{\mathrm{i} 1} & \cdots & \mathrm{u}_{\mathrm{iN}}
\end{array}\right]=\mathrm{S}(0) \mathrm{B}^{\mathrm{i}}, i=0,1,2 .
$$

Finally, to obtain the solution of equation (9) with the conditions (10) by replacing the 3 row matrics (16) by the last 3 rows (or appropriate 3 rows) of the augmented matrix (15) we have the requred augmented matrix

$$
[\tilde{W} ; \tilde{F}]
$$

or clearly

$$
\left[\begin{array}{cccccc}
w_{00} & w_{01} & \ldots & w_{0 N} & ; & f\left(s_{0}\right) \\
w_{10} & w_{11} & \ldots & w_{1 N} & ; & f\left(s_{1}\right) \\
\vdots & \vdots & \vdots & \vdots & \vdots & \vdots \\
w_{N-3,0} & w_{N-3,1} & \cdots & w_{N-3, N} & ; & f\left(s_{N-3}\right) \\
u_{00} & u_{01} & \ldots & u_{0 N} & ; & \lambda_{0} \\
u_{10} & u_{11} & \cdots & u_{1 N} & ; & \lambda_{1} \\
u_{20} & u_{21} & \ldots & u_{2 N} & ; & \lambda_{2}
\end{array}\right]
$$

If $\operatorname{rank} \tilde{W}=\operatorname{rank}[\tilde{W} ; \tilde{F}]=N+1$ then we can write $A=(\tilde{W})^{-1} \tilde{F}$. Thus the matrix A (thereby the coefficients $\left.a_{0}, a_{1}, \ldots, a_{N}\right)$ is uniquely determined. Also equation (9) with the initial conditions (10) has a unique solution. This solution is given by the trancated Taylor series (11). Thus we get the Taylor polynomial solution

$$
Y_{N}(x)=\sum_{n=0}^{N} a_{n} s^{n}
$$

\section{The solution of differential equations characterizing space curves of constant breadth}

We can arrange equation (8) characterizing space curves of constant breadth as follows;

$$
\begin{aligned}
& (\rho \tau)=\mathrm{t} \\
& \mathrm{Q}_{0}(\phi)=-\mathrm{t}^{\prime}(\phi), \mathrm{Q}_{1}(\phi)=\mathrm{t}(\phi)+\mathrm{t}^{3}(\phi), \mathrm{Q}_{2}(\phi)=-\mathrm{t}^{\prime}(\phi), \mathrm{Q}_{3}(\phi)=\mathrm{t}(\phi) \\
& \sum_{\mathrm{k}=0}^{3} \mathrm{Q}_{\mathrm{k}}(\phi) \lambda^{(\mathrm{k})}(\phi)=\mathrm{F}(\phi)
\end{aligned}
$$

Suppose that an approximate solution of this equation (18) for $0 \leq \phi, c \leq 2 \pi$ under the conditions given. This solution has the form of truncated Taylor series.

$$
\lambda(\phi)=\sum_{n=0}^{N} a_{n} \phi^{n}
$$


Here we will take $N=4$ for simplicity. We show the expression (19) in the matrix form as follows;

$$
\lambda(\phi)=S(\phi) A
$$

where $S(\phi)$ and A matrices are defined as $\mathrm{S}(\phi)=\left[\begin{array}{llll}1 & \phi \phi^{2} & \phi^{3} & \phi^{4}\end{array}\right]$ and $\mathrm{A}=\left[\begin{array}{llll}\mathrm{a}_{0} & \mathrm{a}_{1} & \mathrm{a}_{2} & \mathrm{a}_{3} \\ \mathrm{a}_{4}\end{array}\right]^{\mathrm{t}}$. On the other hand $\mathrm{B}, B^{2}$ and $B^{3}$ matrices are defined as follows;

$$
B=\left[\begin{array}{lllll}
0 & 1 & 0 & 0 & 0 \\
0 & 0 & 2 & 0 & 0 \\
0 & 0 & 0 & 3 & 0 \\
0 & 0 & 0 & 0 & 4 \\
0 & 0 & 0 & 0 & 0
\end{array}\right], B^{2}=\left[\begin{array}{ccccc}
0 & 0 & 2 & 0 & 0 \\
0 & 0 & 0 & 6 & 0 \\
0 & 0 & 0 & 0 & 12 \\
0 & 0 & 0 & 0 & 0 \\
0 & 0 & 0 & 0 & 0
\end{array}\right], B^{3}=\left[\begin{array}{ccccc}
0 & 0 & 0 & 6 & 0 \\
0 & 0 & 0 & 0 & 24 \\
0 & 0 & 0 & 0 & 0 \\
0 & 0 & 0 & 0 & 0 \\
0 & 0 & 0 & 0 & 0
\end{array}\right]
$$

for the derivatives $\lambda^{\prime}(\phi)=S(\phi) B A, \quad \lambda^{\prime \prime}(\phi)=S(\phi) B^{2} A$ and $\lambda^{\prime \prime \prime}(\phi)=S(\phi) B^{3} A$. If we put all these expressions in the equation (18), we get following equation

$$
\left\{Q_{3}(\phi) S(\phi) B^{3}+Q_{2}(\phi) S(\phi) B^{2}+Q_{1}(\phi) S(\phi) B+Q_{0}(\phi) S(\phi)\right\} A=F(\phi) .
$$

Now, we use collocation points $\phi=\phi_{i},(i=0,1, \cdots, 4)$ of the specified separation in this equation in the form of matrix

$$
\begin{gathered}
\phi_{0}=0, \phi_{1}=\frac{\pi}{2}, \phi_{2}=\pi, \phi_{3}=\frac{3 \pi}{2}, \phi_{4}=2 \pi \\
Q_{0}(\phi)=\left[\begin{array}{ccccc}
Q_{0}(0) & 0 & 0 & 0 & 0 \\
0 & Q_{0}\left(\frac{\pi}{2}\right) & 0 & 0 & 0 \\
0 & 0 & Q_{0}(\pi) & 0 & 0 \\
0 & 0 & 0 & Q_{0}\left(\frac{3 \pi}{2}\right) & 0 \\
0 & 0 & 0 & 0 & Q_{0}(2 \pi)
\end{array}\right] \\
Q_{1}(\phi)=\left[\begin{array}{ccccc}
Q_{1}(0) & 0 & 0 & 0 & 0 \\
0 & Q_{1}\left(\frac{\pi}{2}\right) & 0 & 0 & 0 \\
0 & 0 & Q_{1}(\pi) & 0 & 0 \\
0 & 0 & 0 & Q_{1}\left(\frac{3 \pi}{2}\right) & 0 \\
0 & 0 & 0 & 0 & Q_{1}(2 \pi)
\end{array}\right] \\
Q_{2}(\phi)=\left[\begin{array}{ccccc}
Q_{2}(0) & 0 & 0 & 0 & 0 \\
0 & Q_{2}\left(\frac{\pi}{2}\right) & 0 & 0 & 0 \\
0 & 0 & Q_{2}(\pi) & 0 & 0 \\
0 & 0 & 0 & Q_{2}\left(\frac{3 \pi}{2}\right) & 0 \\
0 & 0 & 0 & 0 & Q_{2}(2 \pi)
\end{array}\right] \\
Q_{3}(\phi)=\left[\begin{array}{ccccc}
Q_{3}(0) & 0 & 0 & 0 & 0 \\
0 & Q_{3}\left(\frac{\pi}{2}\right) & 0 & 0 & 0 \\
0 & 0 & Q_{3}(\pi) & 0 & 0 \\
0 & 0 & 0 & Q_{3}\left(\frac{3 \pi}{2}\right) & 0 \\
0 & 0 & 0 & 0 & Q_{3}(2 \pi)
\end{array}\right]
\end{gathered}
$$


This matrices can be written briefly as follows;

$$
\begin{aligned}
& Q_{k}(\phi)=\left[\begin{array}{ccccc}
Q_{k}(0) & 0 & 0 & 0 & 0 \\
0 & Q_{k}\left(\frac{\pi}{2}\right) & 0 & 0 & 0 \\
0 & 0 & Q_{k}(\pi) & 0 & 0 \\
0 & 0 & 0 & Q_{k}\left(\frac{3 \pi}{2}\right) & 0 \\
0 & 0 & 0 & 0 & Q_{k}(2 \pi)
\end{array}\right] \\
& S(\phi)=\left[\begin{array}{ccccc}
1 & 0 & 0 & 0 & 0 \\
1 & \left(\frac{\pi}{2}\right) & \left(\frac{\pi}{2}\right)^{2} & \left(\frac{\pi}{2}\right)^{3} & \left(\frac{\pi}{2}\right)^{4} \\
1 & (\pi) & (\pi)^{2} & (\pi)^{3} & (\pi)^{4} \\
1 & \left(\frac{3 \pi}{2}\right) & \left(3 \frac{\pi}{2}\right)^{2} & \left(\frac{3 \pi}{2}\right)^{3} & \left(\frac{3 \pi}{2}\right)^{4} \\
1 & (2 \pi) & (2 \pi)^{2} & (2 \pi)^{3} & (2 \pi)^{4}
\end{array}\right] \\
& F(\phi)=\left[\begin{array}{c}
f(0) \\
f\left(\frac{\pi}{2}\right) \\
f(\pi) \\
f\left(\frac{\pi}{2}\right) \\
f(2 \pi)
\end{array}\right]
\end{aligned}
$$

If we get as

$$
\left\{Q_{3}(\phi) S(\phi) B^{3}+Q_{2}(\phi) S(\phi) B^{2}+Q_{1}(\phi) S(\phi) B+Q_{0}(\phi) S(\phi)\right\}=W
$$

equation (8) is turned to

$$
W A=F \rightarrow[W ; F]
$$

We calculate the W matrix. Then, the equation (17) is written in the form of increased matrix. Furthermore,

$$
\begin{gathered}
\lambda(0)=\lambda_{0} \\
\lambda^{\prime}(0)=\lambda_{1} \\
\lambda^{\prime \prime}(0)=\lambda_{2} .
\end{gathered}
$$

We firstly obtain matrix equation of conditions under the initial conditions given (22) above to achieve an approximate solution of the equation (8).

$$
\begin{gathered}
\lambda(0)=S(0) A=\lambda_{0} \\
\lambda^{\prime}(0)=S(0) B A=\lambda_{1} \\
\lambda^{\prime \prime}(0)=S(0) B^{2} A=\lambda_{2} .
\end{gathered}
$$

So, the expression in the form of increased matrix of matrix equation of conditions is follows as;

$$
\begin{aligned}
& U_{0}=\left[\begin{array}{cccccc}
1 & 0 & 0 & 0 & 0 & ;
\end{array} \lambda_{0}\right] \\
& U_{1}=\left[\begin{array}{llllll}
0 & 1 & 0 & 0 & 0 & ;
\end{array} \lambda_{1}\right] \\
& U_{2}=\left[\begin{array}{llllll}
0 & 0 & 2 & 0 & 0 & ;
\end{array} \lambda_{2}\right]
\end{aligned}
$$


impending,

$$
U=\left[\begin{array}{l}
U_{0} \\
U_{1} \\
U_{2}
\end{array}\right]=\left[\begin{array}{ccccccc}
1 & 0 & 0 & 0 & 0 & ; & \lambda_{0} \\
0 & 1 & 0 & 0 & 0 & ; & \lambda_{1} \\
0 & 0 & 2 & 0 & 0 & ; & \lambda_{2}
\end{array}\right]
$$

Here following equality is obviously.

$$
\mathrm{UA}=\lambda \rightarrow[\mathrm{U} ; \lambda]
$$

We get $W^{*} A=F^{*}$ from (21) and (23) equalities.

$$
\left[\mathrm{W}^{*} ; \mathrm{F}^{*}\right]=\left[\begin{array}{ccccccc}
\mathrm{w}_{00} & \mathrm{w}_{01} & \mathrm{w}_{02} & \mathrm{w}_{03} & \mathrm{w}_{04} ; & \mathrm{f}(0) \\
\mathrm{w}_{10} & \mathrm{w}_{11} & \mathrm{w}_{12} & \mathrm{w}_{13} & \mathrm{w}_{14} ; & \mathrm{f}\left(\frac{\pi}{2}\right) \\
1 & 0 & 0 & 0 & 0 & ; & \lambda_{0} \\
0 & 1 & 0 & 0 & 0 & ; & \lambda_{1} \\
0 & 0 & 2 & 0 & 0 & ; & \lambda_{2}
\end{array}\right]
$$

where, $w_{i j}(i=0,1, j=0,1, \ldots, 4)$ obtained as follows;

$$
\begin{aligned}
& w_{00}=-t^{\prime}(0), w_{01}=t(0)+t^{3}(0), w_{02}=-2 t^{\prime}(0), w_{03}=6 t(0), w_{04}=0, \\
& w_{10}=-t^{\prime}\left(\frac{\pi}{2}\right), w_{11}=-\frac{\pi}{2} t^{\prime}\left(\frac{\pi}{2}\right)+t\left(\frac{\pi}{2}\right)+t^{3}\left(\frac{\pi}{2}\right), \\
& w_{12}=-\frac{\pi}{4} t^{\prime}\left(\frac{\pi}{2}\right)+\pi\left[t\left(\frac{\pi}{2}\right)+t^{3}\left(\frac{\pi}{2}\right)\right]-2 t^{\prime}\left(\frac{\pi}{2}\right) \\
& w_{13}=-\frac{\pi}{8} t^{\prime}\left(\frac{\pi}{2}\right)+\frac{3 \pi^{2}}{4}\left[t\left(\frac{\pi}{2}\right)+t^{3}\left(\frac{\pi}{2}\right)\right]-3 \pi t^{\prime}\left(\frac{\pi}{2}\right)+6 t\left(\frac{\pi}{2}\right) \\
& w_{14}=-\frac{\pi^{4}}{16} t^{\prime}\left(\frac{\pi}{2}\right)+\frac{\pi^{3}}{2}\left[t\left(\frac{\pi}{2}\right)+t^{3}\left(\frac{\pi}{2}\right)\right]-3 \pi^{2} t^{\prime}\left(\frac{\pi}{2}\right)+12 \pi t\left(\frac{\pi}{2}\right)
\end{aligned}
$$

Thus the matrix of unknowns is obtained. $A=W^{*^{-1}} F^{*}$

$$
A=\left[\begin{array}{l}
\lambda_{0} \\
\lambda_{1} \\
\frac{1}{2} \lambda_{2} \\
K \\
M
\end{array}\right]
$$

If we put this $a_{n}$ unknowns in equation (19), we get following equation

$$
\lambda(\phi)=\lambda_{0}+\lambda_{1} \phi+\lambda_{2} \phi^{2}+K \phi^{3}+M \phi^{4}
$$


where $\mathrm{K}$ and $\mathrm{M}$ are 4. and 5. line of matrix A and they are calculated as follows;

$$
\begin{aligned}
K= & \frac{w_{14}}{w_{14} w_{03}-w_{13} w_{04}} f(0)-\frac{w_{04}}{w_{14} w_{03}-w_{13} w_{04}} f\left(\frac{\pi}{2}\right)+\left[-\frac{w_{00}}{w_{03}}+\frac{w_{00} w_{13}+w_{10} w_{03}}{w_{14} w_{03}-w_{13} w_{04}} \frac{w_{04}}{w_{03}}\right] \lambda_{0} \\
& -\left[\frac{w_{01}}{w_{03}}+\frac{w_{01} w_{13}+w_{11} w_{03}}{w_{14} w_{03}-w_{13} w_{04}} \frac{w_{04}}{w_{03}}\right] \lambda_{1}-\frac{1}{2}\left[\frac{w_{02}}{w_{03}}+\frac{w_{02} w_{13}+w_{12} w_{03}}{w_{14} w_{03}-w_{13} w_{04}} \frac{w_{04}}{w_{03}}\right] \lambda_{2} \\
M= & -\frac{w_{13}}{w_{14} w_{03}-w_{13} w_{04}} f(0)+\frac{w_{03}}{w_{14} w_{03}-w_{13} w_{04}} f\left(\frac{\pi}{2}\right)+\left[-\frac{w_{00} w_{13}+w_{10} w_{03}}{w_{14} w_{03}-w_{13} w_{04}}\right] \lambda_{0}+\left[\frac{w_{01} w_{13}+w_{11} w_{03}}{w_{14} w_{03}-w_{13} w_{04}}\right] \lambda_{1} \\
& +\frac{1}{2}\left[\frac{w_{02} w_{13}+w_{12} w_{03}}{w_{14} w_{03}-w_{13} w_{04}}\right] \lambda_{2} .
\end{aligned}
$$

\section{The probed of differential equations characterizing space curves of constant breadth}

We found that the expression is $\lambda$ coefficient which is determined the space curve of constant breadth. $\mu(\phi)$ coefficient is finded with method similar under the same initial conditions. First, it is clear that in the second equation of the system (2)

$$
\lambda=-\mu^{\prime}+\rho \tau \delta
$$

We used where the first equation of the system (2), the derivative of the equation (24)

$$
-\mu^{\prime \prime}+(\rho \tau)^{\prime} \delta+(\rho \tau) \delta^{\prime}=\mu-g
$$

Also, it is clear that in the second equation of the system (2)

$$
\delta=\frac{\mu^{\prime}+\lambda}{\rho \tau}
$$

By using the third equation of the system (2) and the equation (24) in the equation (25), we obtain the following differential equation:

$$
\frac{1}{\rho \tau} \mu^{\prime \prime}+\left(\frac{1}{\rho \tau}\right)^{\prime} \mu^{\prime}+\left(\frac{1}{\rho \tau}\right)^{\prime} \lambda+\frac{(\rho \tau)^{\prime}}{\rho \tau} \mu+\frac{1}{\rho \tau} \mu-\frac{1}{\rho \tau} g=0 .
$$

Here, $\lambda$ is conjugated and then by using derivative of the expression obtained, we obtain the following differential equation;

$$
\lambda^{\prime}=\frac{\rho \tau}{(\rho \tau)^{\prime}} \mu^{\prime \prime \prime}+\left[\left(\frac{\rho \tau}{(\rho \tau)^{\prime}}\right)^{\prime}-1\right] \mu^{\prime \prime}+\left[\rho \tau+\frac{\rho \tau}{(\rho \tau)^{\prime}}\right] \mu^{\prime}+\left[(\rho \tau)^{\prime}+\left(\frac{\rho \tau}{(\rho \tau)^{\prime}}\right)^{\prime}\right] \mu+\left(\frac{\rho \tau}{(\rho \tau)^{\prime}}\right)^{\prime} \mathrm{g}+\frac{\rho \tau}{(\rho \tau)^{\prime}} \mathrm{g}^{\prime}
$$

By using the equality of the equation (27) and the first equation of the system (2) following equation is obtained

$$
\frac{\rho \tau}{(\rho \tau)^{\prime}} \mu^{\prime \prime \prime}+\left[\left(\frac{\rho \tau}{(\rho \tau)^{\prime}}\right)^{\prime}-1\right] \mu^{\prime \prime}+\left[\rho \tau+\frac{\rho \tau}{(\rho \tau)^{\prime}}\right] \mu^{\prime}+\left[(\rho \tau)^{\prime}+\left(\frac{\rho \tau}{(\rho \tau)^{\prime}}\right)^{\prime}-1\right] \mu+\frac{\rho \tau}{(\rho \tau)^{\prime}} \mathrm{g}^{\prime}+\left[\left(\frac{\rho \tau}{(\rho \tau)^{\prime}}\right)^{\prime}+1\right] \mathrm{g}=0
$$

Finally, while $(\rho \tau)=t$ and $\mathrm{F}$ as follows:

$$
F=\frac{\rho \tau}{(\rho \tau)^{\prime}} g^{\prime}+\left[\left(\frac{\rho \tau}{(\rho \tau)^{\prime}}\right)^{\prime}+1\right] g
$$


we obtain the third order, linear, differential equation with variable coefficients as follows

$$
\frac{\mathrm{t}}{\mathrm{t}^{\prime}} \mu^{\prime \prime \prime}+\left[\left(\frac{\mathrm{t}}{\mathrm{t}^{\prime}}\right)^{\prime}-1\right] \mu^{\prime \prime}+\left[\mathrm{t}+\frac{\mathrm{t}}{\mathrm{t}^{\prime}}\right] \mu^{\prime}+\left[\mathrm{t}^{\prime}+\left(\frac{\mathrm{t}}{\mathrm{t}^{\prime}}\right)^{\prime}-1\right] \mu=\mathrm{F} .
$$

This equation is differential equation with unknown $\mu$ characterizing the space curves of constant breadth. Also, $\delta(\phi)$ coefficient is finded with method similar under the same initial conditions. First, it is clear that in the third equation of the system (2)

$$
\mu=-\frac{1}{\rho \tau} \delta^{\prime} .
$$

We used where the second equation of the system (2), the derivative of the equation (30)

$$
\lambda=\frac{1}{\rho \tau} \delta^{\prime \prime}+\left(\frac{1}{\rho \tau}\right)^{\prime} \delta^{\prime}+(\rho \tau) \delta
$$

By using the equation (31) in the first equation of the system (2), we obtain the following differential equation:

$$
\frac{1}{\rho \tau} \delta^{\prime \prime \prime}+2\left(\frac{1}{\rho \tau}\right)^{\prime} \delta^{\prime \prime}+\left[\left(\frac{(\rho \tau)^{\prime}}{(\rho \tau)^{2}}\right)^{\prime}+\frac{1}{\rho \tau}+\rho \tau\right] \delta^{\prime}+(\rho \tau)^{\prime} \delta=g .
$$

Finally, while $(\rho \tau)=t$ we obtain the third order, linear, differential equation with variable coefficients as follows:

$$
\frac{1}{t} \delta^{\prime \prime \prime}+2\left(\frac{1}{t}\right)^{\prime} \delta^{\prime \prime}+\left[\left(\frac{t^{\prime}}{t^{2}}\right)^{\prime}+\frac{1}{t}+t\right] \delta^{\prime}+t^{\prime} \delta=g .
$$

This equation is differential equation with unknown $\delta$ characterizing the space curves of constant breadth.

\section{Corollary}

By using the Taylor matrix method, solution of this equation is approximately obtained as follows:

$$
\mu=\lambda_{0}+\lambda_{1} s+\lambda_{2} s^{2}+P s^{3}+N s^{4} .
$$

We found that the expression is $\mu$ coefficient which is determined the space curve of constant breadth. If these coefficients are written in equation $\lambda^{2}+\mu^{2}+\delta^{2}=R^{2}, \delta$ (or $\mu$ ) will be obtained as

$$
\left(\lambda_{0}+\lambda_{1} s+\lambda_{2} s^{2}+K s^{3}+M s^{4}\right)^{2}+\left(\lambda_{0}+\lambda_{1} s+\lambda_{2} s^{2}+P s^{3}+N s^{4}\right)^{2}+\delta^{2}=R^{2} .
$$

Thus, we obtain general expression connected with torsion and curvature of a space curve of constant breadth.

\section{Competing interests}

The authors declare that they have no competing interests.

\section{Authors' contributions}

All authors have contributed to all parts of the article. All authors read and approved the final manuscript. 
211 B 21 A. Agirman Aydin and M. Sezer: Differential equations characterizing space curves of constant breadth...

\section{References}

[1] Akdoğan, Z., Mağden, A. (2001) Some Characterization of Curves Of Constant Breadth In $E^{n}$ Space, Turk J. Math., 25: 433-444

[2] Dannon, V. (1981) Integral Characterizations And The Theory of Curves, Proc. Amer. Math. Soc., 81, (4): 600-602

[3] Euler L., De Curvis trangularibis, Acta Acad. Petropol., (3-30,(1778),(1780)).

[4] Fujivara, M. (1914) On Space Curves Of Constant Breadth,Thoku Math. J., 5, 179-184

[5] Gluck. H. (1966) Higher Curvatures Of Curves In Euclidean Space, Proc. Amer. Math. Montly, 73 : 699-704

[6] Hacısalihoğlu, H.H. (1993). Diferensiyel Geometri, Ankara Üniversitesi Fen Fakültesi, Ankara, 269s.

[7] Karamete A. (1996) Lineer Diferansiyel Denklemlerin Yaklaşı Çözümü İçin Taylor Stralama Yöntemi, Yüksek Lisans Tezi, Balıkesir Üniversitesi, Balıkesir,43.

[8] Karamete, A.,Sezer, M. (2002) A Taylor collocation method for the solution of linear integro-diferential equations, Int. J. Comput. Math. 79, no.9, 987-1000.

[9] Köse, Ö., Düzlemde Ovaller ve Sabit Genişlikli Eğrilerin bazı özellikleri, Doğa Bilim Dergisi, Seri B, 8(2), 119-126, (1984).

[10] Köse, Ö. (1986) On Space Curve Of Constant Breadth, Doğa TU J. Math., 10, (1): 11-14.

[11] Mağden A., Köse Ö. (1997) On The Curves Of Constant Breadth In E ${ }^{4}$ Space, Turk J. Math., 21: 277-284.

[12] Reuleaux, F. (1963) The Kinematics Of Machinery, Trans. By Kennedy A.B.W., Dover Pub., New York.

[13] Sezer, M. (1989) Differential Equations Characterizing Space Curves Of Constant Breadth And A Criterion For These Curves, Doğa TU J. Math., 13, (2): 70-78.

[14] Sezer, M. (1991) Integral Characterizations For A System of Frenet Like Differential Equations and Applications, E. U. Faculity of Science, Series of Scientific Meetings, no. 1, 435-444. 